\title{
Pathway-specific plasticity in the human spinal cord
}

\author{
Christian Leukel, ${ }^{1,3}$ Wolfgang Taube, ${ }^{3}$ Sandra Beck ${ }^{4}$ and Martin Schubert ${ }^{2}$ \\ ${ }^{1}$ Department of Sport Science, University of Freiburg, Freiburg, Germany \\ ${ }^{2}$ Spinal Cord Injury Centre, University Hospital Balgrist, Zürich, Switzerland \\ ${ }^{3}$ Department of Medicine, Movement and Sport Science, University of Fribourg, Switzerland \\ ${ }^{4}$ Department of Clinical Neurology and Neurophysiology, University of Freiburg, Germany
}

Keywords: cervicomedullary evoked potential, $\mathrm{H}$-reflex conditioning, motor evoked potential, paired associative stimulation, spinal cord, transcranial magnetic stimulation

\begin{abstract}
The aim of the present study was to artificially induce plasticity in the human spinal cord and evaluate whether this plasticity is pathway specific. For this purpose, a technique called paired associative stimulation (PAS) was applied. Volleys evoked by transcranial magnetic stimulation over the primary motor cortex and peripheral nerve stimulation of the nervus tibialis in the popliteal fossa were timed to coincide at the spinal level. The transmission of different corticospinal projections was assessed before and after PAS using conditioned H-reflexes. Different groups of healthy volunteers (28 \pm 5 years) were tested; intervention groups 1 ( $n=9$ ) and $2(n=8)$ received spinal PAS (360 paired stimuli) and the induced effects were evaluated using cortical (group 1) or cervicomedullary (group 2) conditioning of musculus soleus $\mathrm{H}$-reflexes. After spinal PAS, the conditioned H-reflexes were significantly facilitated when tested with cortical and cervicomedullary stimulation. The effect of the latter technique is independent of changes in the excitability of cortical neurons. Therefore, the finding that conditioned H-reflexes were increased after spinal PAS when tested with both cortical and cervicomedullary stimulation suggests that neural plasticity was induced within the spinal cord. The facilitation could only be observed for specific inter-stimulus intervals between volleys induced by peripheral nerve stimulation and transcranial magnetic stimulation. As the specific inter-stimulus intervals were assumed to relate to transmission within specific motor pathways, it is argued that changes in the corticospinal transmission were pathway-specific. These findings may be helpful in inducing and assessing neural plasticity in pathological conditions like spinal cord injuries.
\end{abstract}

\section{Introduction}

Throughout life, the central nervous system is continuously restructured. This restructuring is based on neural plasticity, which can be artificially induced using neurophysiological techniques. Inducing artificial plasticity may help in understanding the mechanisms of plasticity occurring, e.g. while learning a motor task or during recovery from a spinal cord injury (SCI). Improving our understanding of the mechanisms underlying recovery could help to guide the development of treatments for patients who have sustained a SCI. In order to detect, understand, and distinguish the effects of spontaneous and regenerative treatments, it is crucial to develop quantitative assessments of plasticity at the segmental level and of its control by supraspinal input. Ideally, such an approach would allow the quantification of restorative processes in the human spinal cord using non-invasive methods. Transcranial motor cortex stimulation has been used in the past to modify segmental reflexes and thus provided subtle evidence of conduction across the lesion in paralysed subjects with SCI (Alexeeva et al., 1998). Such an approach may allow the assessment of effects from axonal conduction across the lesioned

Correspondence: Christian Leukel, ${ }^{1}$ Department of Sport Science, as above. E-mail: christian.leukel@sport.uni-freiburg.de

spinal cord onto the segmental spinal circuitry, e.g. by probing, through artificially-induced plasticity, the timing and mechanisms of recovery.

A promising model of artificially-induced plasticity is the so-called spike-timing-dependent plasticity originally investigated in animal cell preparations. In this model, pre-synaptic and post-synaptic inputs are applied to a neuron. It was found that the timing of the stimuli determines whether synaptic transmission (changes in the excitability of the associated neurons) was either potentiated (long-term potentiation) or depressed (long-term depression) (Bi \& Poo, 1998; Song et al., 2000). Pre-synaptic excitation preceding post-synaptic activation of the cell caused long-term potentiation, whereas long-term depression was induced when the timing of the stimuli was reversed (Markram et al., 1997). In humans, a similar technique to converge synaptic inputs at cortical neurons and thereby induce plasticity was termed paired associative stimulation (PAS), where the excitation of a peripheral afferent nerve is combined with cortical stimulation to enhance motor cortical excitability (Stefan et al., 2000, 2002; Classen et al., 2004; Kujirai et al., 2006).

Recently, Taylor \& Martin (2009) as well as other groups (Cortes et al., 2011) applied PAS timed to converge two stimuli at the spinal rather than at the cortical level to investigate whether plasticity can also be induced at the $\alpha$-motoneuron pool. Using this approach in the 
study of Taylor \& Martin (2009), antidromic motor axon activation elicited with peripheral nerve stimulation (PNS) was timed to coincide at the $\alpha$-motoneuron pool with motor evoked potentials (MEPs) by transcranial magnetic stimulation (TMS). Changes in the excitability of the spinal motoneurons after application of the PAS paradigm were tested with single-pulse cervicomedullary magnetic stimulation. Different delays between PNS and TMS were used for the PAS intervention so that, in some cases, the corticospinal volley preceded the spinal excitation elicited by PNS and vice versa. The results of this study showed that repeated paired stimuli potentiate or depress corticospinal transmission. However, depression and facilitation did not seem to depend on the sequence of pre-synaptic and post-synaptic excitation as known from spike-timing-dependent plasticity. One reason for this inconsistency may lie in the complexity of the cervicomedullary evoked potential (cMEP). The cMEP is the net sum of all excitatory and inhibitory influences at and downstream of the cervicomedullary level. It lacks temporal resolution concerning the sequence of corticospinal transmission. However, the temporal resolution may be crucial in order to differentiate plasticity effects taking place at specific corticospinal pathways and synapses. Previous studies demonstrated that the MEP (Nielsen et al., 1993; Nielsen \& Petersen, 1995a,b) and also the cMEP (Taube et al., 2011) are composed of a differential sequence of excitatory and inhibitory effects. A time course of facilitation and inhibition can be observed when conditioning the soleus (SOL) H-reflex with either magnetic stimuli of the primary motor cortex (M1, termed "M1 conditioning", in the following) or magnetic stimulation at the cervicomudullary junction (CMJ, termed "CMJ conditioning" in the following) with different inter-stimulus intervals (ISIs). Assuming that the test H-reflex is kept constant, it was previously proposed that different ISIs reflect the excitability of corticospinal pathways with different velocities and different synaptic connections (Nielsen et al., 1993). Thus, effects at early ISIs were attributed to fast, probably monosynaptic corticospinal projections, whereas effects at later ISIs were thought to reflect the excitation of slower and/or indirect pathways. The advantage of this conditioning technique over recording compound muscle potentials such as MEPs and cMEPs is that adaptations in corticospinal transmission can be tested with a high temporal resolution, allowing the identification of sequential inhibitory and facilitatory effects. The purpose of the present study was to identify changes in corticospinal transmission after spinal PAS. We suggested that, using the H-reflex conditioning technique rather than measuring compound muscle action potentials, changes in the transmission can be identified with better temporal precision, allowing a differential analysis of the induced modulation of corticospinal excitability.

\section{Materials and methods General setup}

Twenty-six healthy subjects (aged $27 \pm 5$ years) without neurological or mental disorders participated in the study. The subjects were assigned to two intervention groups. The first intervention consisted of spinal PAS, i.e. TMS was combined with PNS and the stimuli were timed to coincide at the SOL motoneuron pool (see section on Intervention 1). The effect of this intervention was evaluated twofold: (i) using H-reflex conditioning with TMS over the motor cortex (M1 conditioning; $n=9$, group 1), and (ii) using H-reflex conditioning with TMS over the cervicomedullary junction (CMJ conditioning; $n=8$, group 2) (Fig. 1). Cervicomedullary stimulation avoids the direct activation of cortical neurons and therefore provides more direct assessment of neural changes at the spinal level than cortical TMS (Taylor \& Gandevia, 2004). Importantly, note that the H-reflex was conditioned with TMS for both the intervention (spinal PAS, termed intervention in the following), and also as a test to indicate changes in corticospinal excitability before vs. after the intervention (termed pretest and post-test in the following). The procedures are described in detail later (see section on Testing for changes in corticospinal transmission). The second intervention was a control condition in which subjects received low-frequency repetitive TMS (rTMS) over the motor cortex ( $n=9$, group 3) (Fig. 1) at the same frequency and intensity as used during the spinal PAS intervention. The rTMS

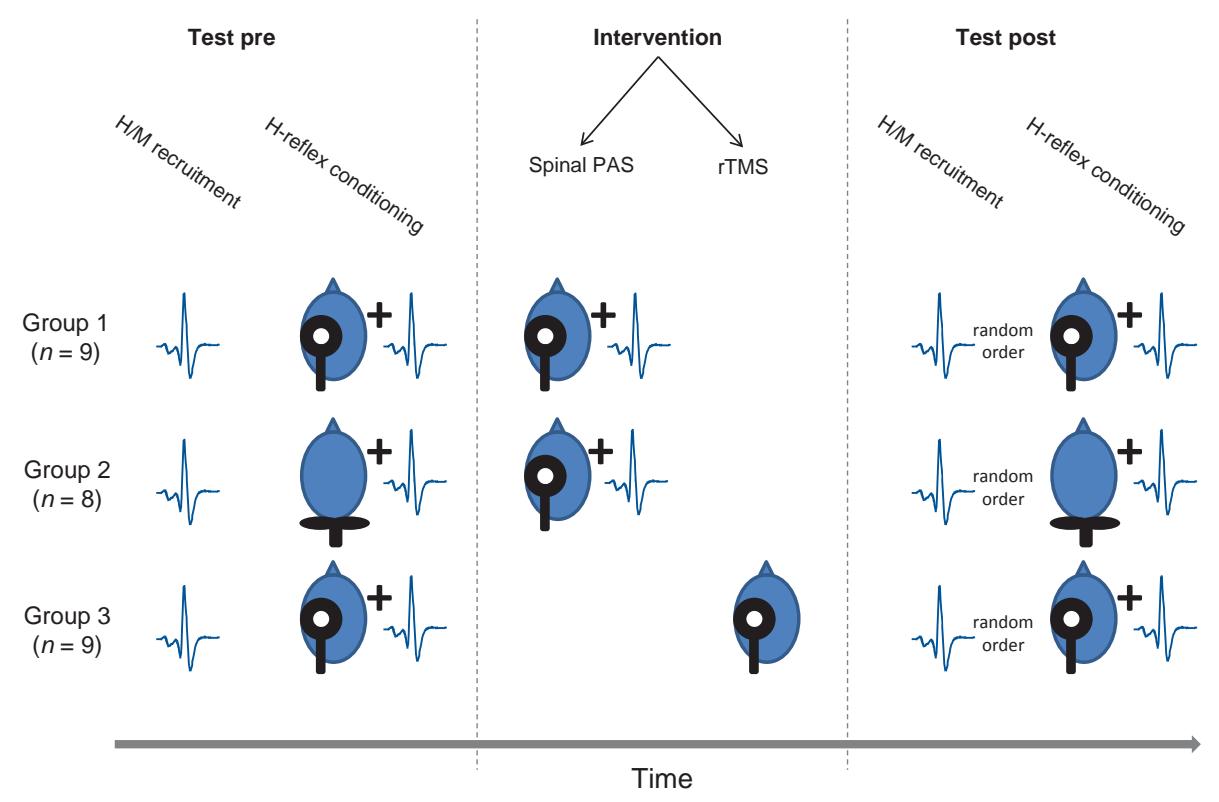

FIG. 1. Overview of the experimental protocol. In all three groups, H-reflex excitability and corticospinal transmission were tested with unconditioned and conditioned H-reflex using either M1 or CMJ stimulation. Thereafter, the intervention (spinal PAS or rTMS) was applied. Finally, H-reflex excitability and corticospinal transmission were tested again. The order of recording $\mathrm{H} / \mathrm{M}$ recruitment curves and conditioned $\mathrm{H}$-reflexes was randomized in the post-test. 
intervention was executed to examine whether changes in corticospinal transmission after spinal PAS cannot simply be explained by the repetitive activation of corticospinal pathways. This was considered to be important as rTMS was shown to induce corticospinal plasticity (Pascual-Leone et al., 1994).

The study was approved by the local ethics committee of the AlbertLudwigs-University in Freiburg and experimental procedures were performed in accordance with the Declaration of Helsinki. All subjects gave written informed consent prior to participation.

\section{Electromyography (EMG)}

The EMG recordings were obtained from the SOL and tibialis anterior muscles of the right leg. After preparation, bipolar surface electrodes (Blue sensor Nl; Ambu ${ }^{\circledR}$, Bad Nauheim, Germany) were attached to the skin longitudinally above the muscle belly $(2 \mathrm{~cm}$ inter-electrode distance). The reference electrode was placed on the tibia plateau. The EMG signals were amplified $(\times 1000)$, bandpass-filtered $(10-1000 \mathrm{~Hz})$ and sampled at $4 \mathrm{kHz}$. The EMG was stored for offline analysis using custom-built software (LabView ${ }^{\circledR}$ based; National Instruments ${ }^{\circledR}$, Austin, TX, USA).

\section{H-reflexes}

The H-reflexes in the right SOL were elicited with an electrical stimulator (constant current stimulator AS100, Alea Solutions ${ }^{\circledR}$, Zürich, Switzerland) by stimulating the posterior tibial nerve in the popliteal fossa. Stimuli consisted of square-wave pulses of $1 \mathrm{~ms}$ duration. The anode, a graphite-coated rubber pad of $5 \times 5 \mathrm{~cm}$, was fixed on the anterior aspect of the knee just underneath the patella. Subjects were seated in a custom-built chair. The cathode $(2 \mathrm{~cm}$ in diameter) was moved stepwise until the optimal position for eliciting an H-reflex was found (the time interval between successive stimuli was $5 \mathrm{~s}$ ). Stimulation intensities ranged from 5 to $15 \mathrm{~mA}$. Care was taken that the stimulation did not activate the common peroneal nerve as tested with parallel recordings from the tibialis anterior. After the optimal position was found, the cathode (Blue sensor N, Ambu ${ }^{\circledR}$ ) was fixed with tape.

\section{Transcranial magnetic stimulation}

The MEPs in the SOL were elicited by TMS of the contralateral motor cortical leg area using a MagVenture ${ }^{\circledR}$ magnetic stimulator (MagPro X 100, Farum, Denmark) with the capability to deliver a magnetic field of $1.5 \mathrm{~T}$ for $200 \mu$ s through a figure-of-eight coil (MC-B70). After positioning the coil over the SOL hotspot of the cortical leg area, the resting motor threshold (MT) (1.0 MT) was determined (induced current was posterior-anterior). The MT was defined as the intensity of magnetic stimulation required to evoke MEPs of $50 \mu \mathrm{V}$ peak-topeak amplitude in at least three of five consecutive trials. 1.0 MT was expressed as a percentage of the maximum stimulator output. To ensure a constant position of the coil throughout the experiment, the head of the subjects and the coil were mechanically fixed. Additionally, the position was marked on the scalp with a waterproof marker

\section{Intervention 1: spinal paired associative stimulation}

In order to induce (spinal) plasticity, subjects received 360 paired stimuli with a frequency of $0.2 \mathrm{~Hz}$, i.e. the afferent volley by PNS collided 360 times with the evoked volleys by TMS at the level of the spinal motoneurons (TMS was again elicited by the MagVenture ${ }^{\circledR}$ magnetic stimulator MagPro X 100). TMS was triggered after a delay of $1 \mathrm{~ms}$ (ISI -1) with respect to PNS. The reason for using this specific ISI was to ensure that conditioning took place at the spinal level with the Ia afferent volley arriving at the spinal motoneuron after this had just been activated by the earliest component of the TMSinduced corticospinal volley. The reason for this has been shown by demonstrating that, with simultaneous TMS and PNS (ISI $=0$ ), the fastest (probably monosynaptic) corticospinal volleys usually arrive at the spinal $\alpha$-motoneurons at $2-5 \mathrm{~ms}$ prior to the Ia afferent volley elicited by PNS (Nielsen et al., 1993; Taube et al., 2006; Schubert et al., 2008). The typical ISI to obtain the earliest facilitation of the $\mathrm{H}-$ reflex with TMS conditioning varied between -2 and $-5 \mathrm{~ms}$ because of inter-individual differences in physiological and anatomical parameters of the subjects. In other words, between ISI -2 and $-5 \mathrm{~ms}$, the initial part of the corticospinal volley and the afferent Ia volley coincide at the spinal motoneurons. This means, e.g. when an ISI of $-3 \mathrm{~ms}$ is used for the spinal PAS intervention, that it might be possible that the volleys by PNS and TMS would not coincide at the $\alpha$ motoneurons in some subjects. Thus, an ISI of $-1 \mathrm{~ms}$ was used for the spinal PAS intervention to ensure the coincidence of volleys at the spinal motorneuron in all subjects. It is possible that spinal interneurons are also activated by the volleys converging at the spinal level but, based on the timing, not supraspinal neurons. The intensity for the electrical stimulation was set to elicit H-reflexes with a size of $15-25 \%$ of the maximum M-wave $\left(M_{\max }\right)$ (Crone et al., 1990). The stimulation intensity for TMS was set to 1.0 MT. A total of 17 subjects received combined TMS and H-reflexes (spinal PAS), and were either tested with M1 or CMJ conditioning. During the entire experiment, all subjects were instructed to close their eyes and maintain the same relaxed position while sitting in a custom-built chair.

\section{Intervention 2: repetitive transcranial magnetic stimulation}

To control for corticospinal plasticity induced by rTMS of M1, nine subjects participated in Intervention 2. The subjects received rTMS with a frequency of $0.2 \mathrm{~Hz}$ and were tested with M1 conditioning of the SOL H-reflex before and after the intervention.

\section{Testing for changes in corticospinal transmission}

\section{M1 conditioning}

The conditioning technique, which was used to test plasticity after Intervention 1 (spinal PAS) and Intervention 2 (rTMS), was applied in accordance with previous studies (Nielsen et al., 1993; Nielsen \& Petersen, 1995a,b; Petersen et al., 1998; Schubert et al., 2008). PNS with an intensity to evoke SOL H-reflexes of $15-25 \%$ of the $M_{\max }$ (Crone et al., 1990) and TMS with an intensity of 1.0 MT were combined at different ISIs $(-5,-4,-3,-2,-1,0,1 \mathrm{~ms})$. Negative ISIs (in ms) indicate that the electrical stimulus was given before TMS. The reason for starting with an ISI of $-5 \mathrm{~ms}$ was to ensure the measurement of the early facilitation of the conditioned H-reflexes. The early facilitation can be observed when PNS precedes TMS by $2-$ $4 \mathrm{~ms}$ and probably indicates activation of the spinal motoneurons by the fastest (monosynaptic) corticospinal fibres (Nielsen et al., 1993). The stimulation intensities for PNS and TMS were set before recording the H-reflex conditioning curves in the pre-test. The stimulation intensity for TMS was retained for the H-reflex conditioning curves after spinal PAS and after rTMS in the post-test. The stimulation intensity for PNS was adjusted in the post-test so that the size of the unconditioned H-reflex in the post-test matched the size of the unconditioned H-reflex in the pre-test. Each ISI was measured 10 
times in a randomized order including the same number of unconditioned H-reflexes and MEPs induced by single-pulse TMS. The time interval between successive stimuli was $5 \mathrm{~s}$ (stimulation frequency of $0.2 \mathrm{~Hz}$ ).

\section{CMJ conditioning}

Cervicomedullary stimulation was applied to assess changes in transmission of corticospinal pathways in response to spinal PAS. In contrast to cortical (M1) magnetic stimulation, the activation of $\alpha$ motoneurons by cervicomedullary stimulation does not depend, at least for the first part of the descending corticospinal volleys (Ugawa et al., 1997), on changes in the excitability of cortical neurons. Thus, changes shown with CMJ conditioning of the SOL H-reflex following spinal PAS can probably be explained by adaptations at the spinal level. Due to the shorter travel distance between the stimulation site and activated muscle, the latency of the corticospinal volley is 3-4 ms shorter when TMS is applied at the cervicomedullary junction compared with TMS of the motor cortex (Taylor \& Gandevia, 2004; Taylor et al., 2006). Therefore, the following ISIs for CMJ conditioning were used in the present study: $-9,-8,-7,-6,-5$ and $-4 \mathrm{~ms}$. PNS was elicited with an intensity to evoke SOL H-reflexes of 15$25 \%$ of the $M_{\max }$ (Crone et al., 1990). Cervicomedullary magnetic stimulation was always applied with maximum stimulator output using a Magstim ${ }^{\circledR}$ magnetic stimulator (Magstim Rapid, Wales, UK) with a double-cone coil. The stimulation intensity for PNS was set before recording the H-reflex conditioning curves in the pre-test. This intensity was adjusted in the post-test so that the size of the unconditioned H-reflex in the post-test matched the size of the unconditioned H-reflex in the pre-test. Each ISI was measured 10 times in a randomized order including the same number of unconditioned H-reflexes and cMEPs induced by single-pulse cervicomedullary stimulation. The time interval between successive stimuli was $5 \mathrm{~s}$ (stimulation frequency of $0.2 \mathrm{~Hz}$ ).

The double-cone coil for cervicomedullary stimulation was placed in the same way as described previously for stimulating the corticospinal tract (Taylor \& Gandevia, 2004) and the subjects sat in the custom-built chair. When CMJ conditioning was performed, the subjects were asked to bend their head and back forward in order to position the magnetic coil and therefore the magnetic field closer to the corticospinal tract.

\section{Experimental procedure}

All measurements were performed at rest. After the subjects sat comfortably and relaxed, maximum H-reflex (Hmax)/maximum M-wave (Mmax) (H/M) recruitment curves were recorded and M1 or CMJ conditioning of the SOL H-reflex was performed. Thereafter, the spinal PAS or the rTMS intervention was applied. After the 360 combined (spinal PAS) or single TMS (rTMS) stimuli, the SOL H-reflex was again conditioned with cortical TMS or cervicomedullary TMS as soon as the setup was ready (approximately $1 \mathrm{~min}$ after the last stimulus of spinal PAS/rTMS was delivered). The $\mathrm{H} / \mathrm{M}$ recruitment curves were recorded. $\mathrm{M} 1$ or $\mathrm{CMJ}$ conditioning and recording of $\mathrm{H} / \mathrm{M}$ recruitment curves were carried out in a pseudo-randomized order (see Fig. 1).

\section{Data analysis and statistics}

The MEPs, unconditioned H-reflexes, conditioned H-reflexes and M-waves were expressed as peak-to-peak amplitudes of the unrectified EMG. Before and after the interventions (spinal PAS or rTMS), testing with M1 and CMJ conditioning took place where 10 MEPs, 10 conditioned H-reflexes (at each ISI), and 10 control (unconditioned) H-reflexes were recorded and subsequently averaged. The control (unconditioned) MEPs (compound potentials) were additionally analysed to compare the current findings with previous (cortical) PAS studies (Stefan et al., 2000, 2002). The control (unconditioned) $\mathrm{H}$-reflexes served as a reference for the conditioned H-reflexes. The intra-individual mean of the conditioned H-reflex (at each ISI) was divided by the intra-individual mean of the unconditioned control Hreflex. In order to elucidate neural plasticity after spinal PAS and rTMS by means of the conditioned H-reflexes, the individual onset of the 'early facilitation' was determined (Nielsen et al., 1993). The early facilitation denominates the ISI where a facilitation of the conditioned H-reflex is first detectable (with respect to the delay between TMS and PNS). It was suggested that this facilitation indicates the coincidence of the PNS evoked (Ia) afferent volley at the spinal motoneurons with the fastest, presumably monosynaptic, corticospinal volley (Nielsen et al., 1993). There is an individual variability in the onset of the early facilitation caused by variations of the anatomical and physiological parameters of the subjects (e.g. trunk length, leg length), which results in differences in conduction times of the corticospinal and (Ia) afferent volley to reach the spinal $\alpha$-motoneurons. Therefore, synchronization to the individual early facilitation was necessary for a valid comparison of effects across subjects. To detect the individual early facilitation, the conditioned H-reflexes recorded at each ISI in the pretest were compared with the control H-reflexes using non-parametric Wilcoxon tests. The ISI corresponding to the first significantly facilitated conditioned H-reflexes indicated the onset of the early facilitation. In each subject, the duration of this early facilitation was around 1-2 ms and was followed by a drop in the size of the conditioned H-reflexes (Nielsen et al., 1993), which allowed a clear detection of the ISI representing the early facilitation.

The H/M recruitment curves were plotted from approximately 30 60 stimuli ranging from the $\mathrm{H}$-reflex threshold $(500 \mu \mathrm{V}$ peak-to-peak amplitude) to the intensity to evoke $M_{\max } . H_{\max }$ and $M_{\max }$ were determined as the largest single response from the peak-to-peak amplitudes of the unrectified SOL EMG, and $\mathrm{H} / \mathrm{M}$ ratios were calculated.

All variables are expressed as mean \pm SD. Before statistical comparison, all data sets were tested for normal distribution by a Kolmogorov-Smirnov test. Normalized conditioned H-reflex amplitudes recorded in the pre-test and post-test were analysed by means of repeated-measures ANOVA with the within-subject factor time (before vs. after spinal PAS), and the between-subject factor test group (M1 vs. CMJ conditioning). The conditioned $\mathrm{H}$-reflexes obtained in the rTMS group were analysed by means of repeated-measures ANOVA with the within-subject factor time. H-reflexes and M-waves for groups with spinal PAS were analysed using a repeated-measures ANOVA with the within-subject factor time and the between-subject factor test group (M1 vs. CMJ conditioning). H-reflexes and M-waves in the rTMS group were analysed with paired Student's $t$-tests. Paired Student's $t$-tests were also used to indicate differences between conditioned H-reflexes of the pre-test and post-test at each ISI for each intervention (spinal PAS and rTMS). Differences were regarded as significant at $P<0.05$ for all tests. SPSS software 15.0 (SPSS $^{\circledR}$, Chicago, IL, USA) was used for the statistical tests.

\section{Results}

\section{Intervention 1: spinal paired associative stimulation}

Changes in the corticospinal transmission after the spinal PAS intervention were tested by either M1 or CMJ conditioning. The 
conditioned H-reflexes were synchronized with respect to the individual early facilitation (see Materials and methods) and grand mean values were calculated for each ISI based on the synchronized data. Conditioned H-reflex amplitudes were analysed for the following six ISIs: the early facilitation (termed $\mathrm{f} 1$ for $\mathrm{M} 1$ conditioning and $\mathrm{CMJ}$ conditioning in the following), the ISI preceding $\mathrm{f} 1$, which was tested at $1 \mathrm{~ms}$ prior to the early facilitation (termed f0), and four ISIs, which followed the early facilitation at intervals of $1 \mathrm{~ms}$ (termed f2, f3, f4, and f5, respectively).

The conditioned H-reflexes significantly differed with respect to the factor time $\left(F_{1,2}=20.61, P=0.045\right)$ but not between groups (i.e. $\mathrm{M} 1$ and $\mathrm{CMJ}$ conditioning yielded the same result; group: $F_{1,2}=3.30, P=0.21$, Figs 3 and 4 ). The early facilitation occurred at $-3.0 \pm 0.9 \mathrm{~ms}$ (group mean values) for the subjects with M1 conditioning. An early facilitation at approximately $-3 \mathrm{~ms}$ was also reported in previous studies (Nielsen \& Petersen, 1995a,b; Nielsen et al., 1993). The early facilitation for subjects with CMJ conditioning occurred at $-7.1 \pm 0.8 \mathrm{~ms}$. Post-hoc analysis of the conditioned H-reflexes of the group with M1 conditioning yielded significantly higher amplitudes in the post-intervention compared with the pre-intervention at $\mathrm{f} 3(P=0.03)$, corresponding to an ISI of

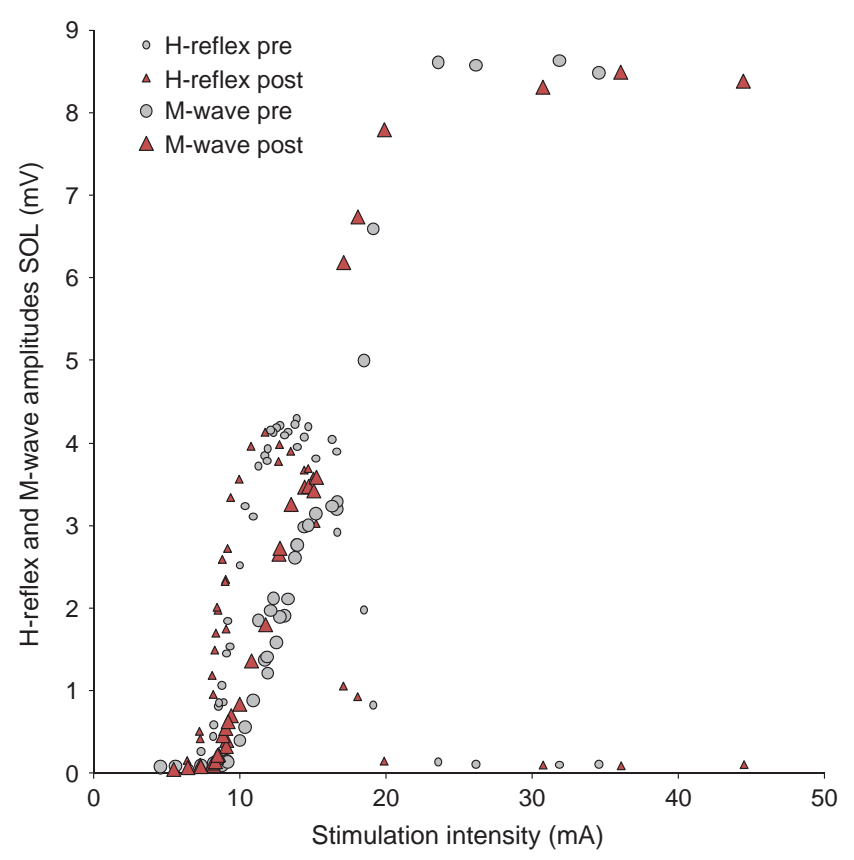

FIG. 2. H/M recruitment curves of an individual subject recorded before (pre) and after (post) the spinal PAS protocol.
$-1 \mathrm{~ms}$ within the synchronized grand mean data set. Furthermore, post-intervention values were higher at $\mathrm{f} 4(P=0.04$; corresponding to an ISI of $0 \mathrm{~ms}$ ) and f5 ( $P=0.01$; corresponding to an ISI of $1 \mathrm{~ms}$, Fig. 3). Post-hoc analysis of CMJ conditioning indicated that conditioned H-reflex amplitudes were higher in the post-test than in the pre-test at $\mathrm{f3}(P=0.03)$ and $\mathrm{f5}(P=0.01)$, corresponding to ISIs of $-5 \mathrm{~ms}$ and $-3 \mathrm{~ms}$ of the synchronized grand mean data set, respectively.

The grand mean values of SOL MEP amplitudes recorded with M1 conditioning were significantly increased after spinal PAS (pre-test: $89 \pm 42 \mu \mathrm{V}$; post-test: $131 \pm 87 \mu \mathrm{V}, P=0.04$ ). The $H_{\max }$ and $M_{\max }$ were not significantly different with respect to the factors time and group (Fig. 2, Table 1).

\section{Intervention 2: repetitive transcranial magnetic stimulation}

The conditioned H-reflexes did not differ with respect to the factor time $\left(F_{1,1}=0.88, P=0.52\right.$, Fig. 3$)$. The early facilitation (group mean values) occurred at $-2.0 \pm 0.76 \mathrm{~ms}$. Post-hoc analysis of the conditioned H-reflexes revealed significantly lower values in the posttest compared with the pre-test at $\mathrm{f} 1(P=0.001$; corresponding to an ISI of $-2 \mathrm{~ms}$ of the synchronized grand mean data set) and f3 $(P=0.03$; corresponding to an ISI of $0 \mathrm{~ms}$ of the synchronized grand mean data set) (Fig. 3). There were no significant changes of the MEP amplitudes (pre-test: $99 \pm 42 \mu \mathrm{V}$; post-test: $88 \pm 29 \mu \mathrm{V}, P=0.58$ ).

The $M_{\max }$ did not differ between the pre-test and post-test, whereas $H_{\max }$ was significantly reduced $\left(H_{\max }: P=0.01 ; M_{\max }: P=0.12\right.$, Table 1).

\section{Discussion}

The main finding of the present study was an increase of the size of the conditioned H-reflex at the targeted ISI following the spinal PAS intervention. This facilitation was shown with both M1 and CMJ conditioning, whereas no changes were observed for $H_{\max }$ and $M_{\max }$. Consistent $M_{\max }$ amplitudes in the pre-test and post-test indicate that neither the stimulation nor the recording conditions were altered. Consistent $H_{\max }$ amplitudes in the pre-test and post-test indicate that the excitability of the H-reflex pathway was not modified by spinal PAS, suggesting that this spinal reflex circuit underwent no substantial changes that could account for the adaptations observed for the conditioned H-reflex following spinal PAS. Therefore, the facilitation of the conditioned H-reflexes may most likely be explained by the strengthening of corticospinal transmission. As the effects were similar with $\mathrm{M} 1$ and $\mathrm{CMJ}$ conditioning, and as CMJ conditioning bypasses cortical cells, which may have a change in excitability after the spinal PAS intervention, it is argued that plasticity was, in all likelihood, induced at the spinal level.

TABLE 1. Mean values $( \pm \mathrm{SD})$ of $H_{\max }$ and $M_{\max }$ in $\mathrm{mV}$

\begin{tabular}{|c|c|c|c|c|}
\hline \multirow[b]{2}{*}{ Group } & \multicolumn{2}{|c|}{ Pre-intervention } & \multicolumn{2}{|c|}{ Post-intervention } \\
\hline & $H_{\max }$ & $M_{\max }$ & $H_{\max }$ & $M_{\max }$ \\
\hline Spinal PAS - cortical conditioning & $4.88 \pm 3.65$ & $9.57 \pm 3.78$ & $4.50 \pm 3.94$ & $8.96 \pm 3.91$ \\
\hline Spinal PAS - cervicomedullary conditioning & $6.65 \pm 2.45$ & $10.19 \pm 3.45$ & $7.02 \pm 2.55$ & $10.24 \pm 4.02$ \\
\hline rTMS - cortical conditioning & $8.83 \pm 4.49$ & $12.93 \pm 5.39$ & $7.74 \pm 5.01$ & $12.20 \pm 5.27$ \\
\hline
\end{tabular}

Pre-intervention refers to the values before the intervention (spinal PAS or rTMS) and post-intervention refers to the values after the intervention. For reasons of clarity, levels of significance are reported in the text. 

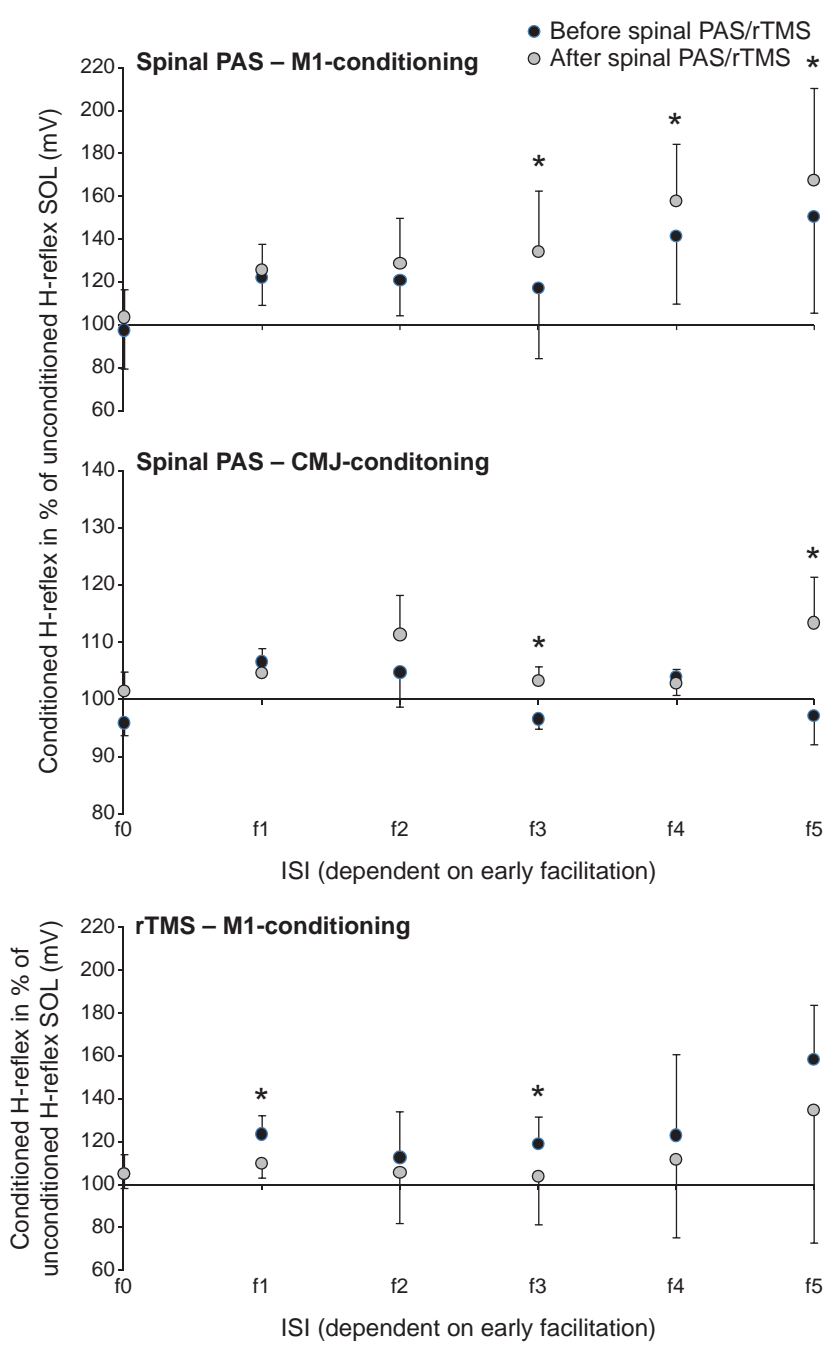

FIG. 3. Normalized (based on the individual onset of the early facilitation) grand mean curves of the conditioned H-reflex of all three tested groups (spinal PAS with M1 and CMJ conditioning, rTMS with M1 conditioning). The ISI indicating the onset of the early facilitation is named $\mathrm{f} 1$. $\mathrm{f} 0$ is $1 \mathrm{~ms}$ prior to the early facilitation and $\mathrm{f} 2$ is $1 \mathrm{~ms}$ after $\mathrm{f} 1$ ( $\mathrm{f} 3, \mathrm{f} 4$ and $\mathrm{f} 5$ are also separated by $1 \mathrm{~ms}$, respectively). Black circles indicate the conditioned H-reflexes in the pretest, grey circles show the conditioned H-reflexes in the post-test. Black bars indicate SD. Asterisks represent significant differences between the pre-test and post-test.

\section{Evidence for spinal plasticity}

The H-reflex pathway remained unchanged after spinal PAS, as was indicated by unaltered $\mathrm{H} / \mathrm{M}$ recruitment curves. The facilitated conditioned H-reflexes therefore have to be caused by changes in corticospinal transmission somewhere between the stimulation site and the $\alpha$-motoneurons in the spinal cord. When tested with M1 conditioning, corticospinal volleys could have been altered at the cortical or spinal level. Based on the rTMS data showing a decrease of corticospinal excitability, an isolated cortical facilitation from repetitive cortical stimulation seems unlikely to be responsible for the increase of the conditioned H-reflexes after spinal PAS. The effect of repetitive peripheral stimulation alone has not been assessed as an intervention in the current study. However, previous studies looking at the influence of repetitive afferent stimulation (Cortes et al., 2011) have not shown substantial changes that could explain the currently observed adaptations in corticospinal transmission. Consequently, the convergence of afferent volleys induced by PNS and descending volleys elicited by TMS seems to be crucial for the effects on conditioned H-reflexes seen in the present study. The first ISI that was significantly facilitated after the spinal PAS intervention with M1 conditioning was ISI $\mathrm{f} 3$, corresponding to an ISI of $-1 \mathrm{~ms}$ in the synchronized grand mean data set. Thus, the facilitation started at exactly the ISI that was used for the spinal PAS intervention. During the 30 min spinal PAS intervention, the H-reflex was always elicited at $1 \mathrm{~ms}$ before the TMS pulse (ISI of $-1 \mathrm{~ms}$ ). Consequently, it seems reasonable that the transmission of the corticospinal pathway(s) activated during the intervention adapted in response to spinal PAS.

Although it seems unlikely that cortical adaptations are responsible for the facilitations of the conditioned H-reflexes observed following spinal PAS, assessment of the corticospinal transmission by means of H-reflex conditioning with TMS over the M1 cannot completely exclude cortical plasticity. Therefore, a second testing method was applied where changes at the cortical level could be discarded - at least for the ISIs tested in the present study. For this purpose, cervicomedullary stimulation was used to assess changes in corticospinal transmission.

After the spinal PAS intervention, H-reflexes conditioned with cervicomedullary stimulation were significantly augmented after spinal PAS. A first significant PAS-associated increase of the conditioned H-reflex started at $\mathrm{f}$, corresponding to an ISI of $-5 \mathrm{~ms}$ of the synchronized grand mean data set. This means that, following the spinal PAS intervention, the earliest effects occurred when the H-reflex was elicited on average $5 \mathrm{~ms}$ before the cervicomedullary stimulation. Independent of spinal PAS intervention, the onset of an early facilitation with CMJ conditioning was observed at an ISI $2 \mathrm{~ms}$ earlier, i.e. at $-7 \mathrm{~ms}$ (f1). Thus, a significant PAS-associated increase of the conditioned H-reflex occurred $2 \mathrm{~ms}$ later than the onset of early H-reflex facilitation that was observed with both testing methods (corresponding to $\mathrm{f} 3$ for both $\mathrm{M} 1$ and $\mathrm{CMJ}$ conditioning). Assuming that, independent of the testing method (M1 and CMJ conditioning), the early facilitation is caused by activity in the fastest, probably direct corticospinal pathways, it could be argued that spinal PAS may specifically influence the corticospinal transmission at a spinal level, possibly at the corticospinal synapse(s). However, although the present results suggest that plasticity occurred at the spinal level, the exact site of action is not yet clear. Neural changes might have occurred at synapses between direct and/or indirect corticospinal fibres and the $\alpha$-motoneurons. Alternatively, the excitability of propriospinal neurons connecting corticospinal fibres with $\alpha$-motoneurons might have changed. Changes of the H-reflex pathway, including modulations of the pre-synaptic inhibition of Ia afferent fibres shown after cortical PAS (Lamy et al., 2010), seem less likely. In contrast to studies applying cortical PAS (Meunier et al., 2007; Lamy et al., 2010), the excitability of the H-reflex and M-wave did not change after spinal PAS in the present study.

\section{Methodological considerations}

Compared with the analysis of compound potentials, conditioning of the H-reflex provides the advantage of assessing corticospinal transmission with a high temporal resolution. This has been proposed to allow the distinction of different corticospinal projections (Nielsen et al., 1993; Nielsen \& Petersen, 1995a,b; Schubert et al., 2008; Taube et al., 2011). Distinct excitatory and inhibitory effects in corticospinal transmission can be evaluated, which is not obvious from the compound potentials, where all excitatory and inhibitory influ- 


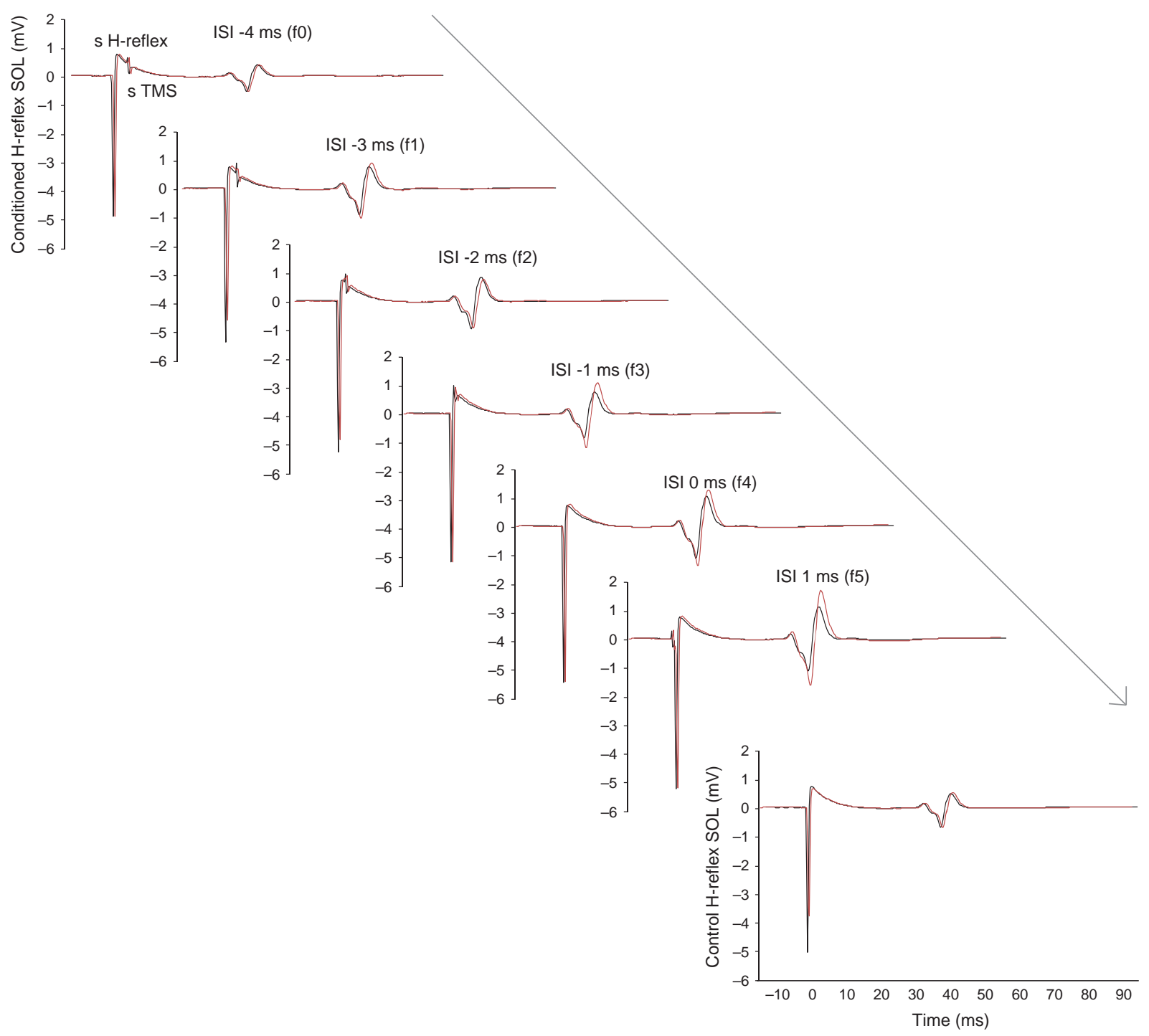

FIG. 4. M1 conditioning before and after spinal PAS of an individual subject. Each trace represents the mean of 10 recorded sweeps. Displayed are the control (unconditioned) H-reflexes as well as the conditioned H-reflexes for ISIs ranging from $-4 \mathrm{~ms}$ to $1 \mathrm{~ms}$ recorded in the pre-test (black traces) and post-test (red traces). The stimulus artefact for the electrical stimulation is marked as 's H-reflex'. The stimulus artefact for TMS is named 's TMS'. Note that the onset of the early facilitation (f1) in this subject is at an ISI of $-3 \mathrm{~ms}$. The facilitation of the conditioned H-reflexes after spinal PAS starts at an ISI of $-1 \mathrm{~ms}$ (f3).

ences summate. Moreover, conditioning of the H-reflex at the CMJ allows the assessment of responses to cervicomedullary stimulation in the resting state, which is usually not possible, e.g. in muscles of the lower limb. This is due to the afferent volley elicited by PNS providing a standardized 'baseline' excitation at the spinal motoneurons, which are consequently (i) at a similar excitability level, and (ii) more susceptible to the excitation transmitted via the corticospinal tract following cervicomedullary stimulation. Accordingly, there may be a reduced risk of overlooking effects than when analysing compound potentials (Taylor \& Martin, 2009). With compound potentials, changes in the corticospinal transmission (specifically simultaneously altered excitatory and inhibitory influences) could be balanced and therefore the resulting MEP/cMEP would be unchanged in spite of ongoing adaptations. Furthermore, measuring compound potentials at rest inherits the limitation that the excitability level of the $\alpha$-motoneurons cannot be controlled and, thus, changes observed might reflect fluctuations in the excitability level of the motoneuron pool after the PAS intervention.
Cortes et al. (2011) used another ISI in a spinal associative stimulation protocol with subthreshold corticospinal tract stimulation by TMS preceding posterior tibial nerve stimulation by $20 \mathrm{~ms}$ to modulate the H-reflex. In contrast, we used an ISI of $-1 \mathrm{~ms}$ in the present study. At this ISI, we argued that volleys by TMS and PNS coincide at the spinal motoneurons in all subjects. Later, positive ISIs mean that TMS is elicited earlier than PNS, as in the study of Cortes et al. (2011). Consequently, at these ISIs, the volley induced by PNS may have sufficient time to reach supraspinal structures and thereby coincide with volleys by TMS. In other words, the probability of inducing (additional) cortical plasticity by coinciding volleys with TMS and PNS is higher when using large positive ISIs. Cortes et al. (2011) reported strong modulations of the maximum H-reflex amplitude, unobserved in our study, thus indicating that the timing of stimuli is crucial for PAS-induced effects. Although these authors also suggested that the descending corticospinal activity interacts with the afferent peripheral activity at the spinal cord level (Valls-Sole et al., 1994), they were not able to pinpoint the possible involvement 
of distinct corticospinal projections. These, however, could be most important for motor recovery after incomplete spinal injury (Curt et al., 2004). As shown by a much earlier study by Alexeeva et al. (1998), applying TMS conditioning of the SOL H-reflex in incomplete SCI to investigate the delay in the latency of TMS-induced MEPs, the earliest indication of a TMS effect on H-reflex excitability in subjects with SCI was delayed by $10 \mathrm{~ms}$ as compared with healthy subjects. Therefore, the thorough investigation of plasticity effects by extensive testing of ISI as put forward in the present study is paramount to provide a sufficiently high time resolution to detect and correctly attribute plasticity effects.

A drawback of the H-reflex conditioning technique with TMS/ cervicomedullary stimulation is that it is time-consuming. As a consequence, it was not possible to test several different ISIs between TMS and PNS for the spinal PAS intervention as was done in the study of Taylor \& Martin (2009). The present study had to focus on one specific ISI for the intervention.

\section{Functional considerations}

An important issue related to neural plasticity is behavioural outcome, e.g. in the rehabilitation process after incomplete SCI. Many studies have described the relevance of spinal plasticity for motor function. Corticospinal connections were especially argued to drive these plastic changes (Wolpaw \& Kaas, 2001; Wolpaw, 2010). Consequently, the method presented in this study could have the potential not only to improve assessment but also to influence the outcome of training and rehabilitation, e.g. following spinal injury as has been shown in several recent pivotal studies (Roy et al., 2010; Thompson et al., 2011).

\section{Acknowledgements}

This study was funded by a grant from the Betty und David Koetser Stiftung für Hirnforschung (Zürich, Switzerland).

\section{Abbreviations}

cMEP, cervicomedullary evoked potential; CMJ, cervicomedullary junction; EMG, Electromyography; $\mathrm{H} / \mathrm{M}, H_{\max } / M_{\max } ; \mathrm{H}_{\max }$, maximum H-reflex; ISI, inter-stimulus interval; $\mathrm{M} 1$, primary motor cortex; $\mathrm{M}_{\max }$, maximum M-wave; MEP, motor evoked potential; $M_{\max }$, maximum M-wave; MT, motor threshold; PAS, paired associative stimulation; PNS, peripheral nerve stimulation; rTMS, repetitive transcranial magnetic stimulation; SCI, spinal cord injury; SOL, soleus; TMS, transcranial magnetic stimulation.

\section{References}

Alexeeva, N., Broton, J.G. \& Calancie, B. (1998) Latency of changes in spinal motoneuron excitability evoked by transcranial magnetic brain stimulation in spinal cord injured individuals. Electroencephalogr. Clin. Neurophysiol., 109, 297-303.

Bi, G.Q. \& Poo, M.M. (1998) Synaptic modifications in cultured hippocampal neurons: dependence on spike timing, synaptic strength, and postsynaptic cell type. J. Neurosci., 18, 10464-10472.

Classen, J., Wolters, A., Stefan, K., Wycislo, M., Sandbrink, F., Schmidt, A. \& Kunesch, E. (2004) Paired associative stimulation. Suppl. Clin. Neurophysiol., 57, 563-569.

Cortes, M., Thickbroom, G.W., Valls-Sole, J., Pascual-Leone, A. \& Edwards, D.J. (2011) Spinal associative stimulation: a non-invasive stimulation paradigm to modulate spinal excitability. Clin. Neurophysiol., 122, 22542259.

Crone, C., Hultborn, H., Mazieres, L., Morin, C., Nielsen, J. \& PierrotDeseilligny, E. (1990) Sensitivity of monosynaptic test reflexes to facilitation and inhibition as a function of the test reflex size: a study in man and the cat. Exp. Brain Res., 81, 35-45.
Curt, A., Schwab, M.E. \& Dietz, V. (2004) Providing the clinical basis for new interventional therapies: refined diagnosis and assessment of recovery after spinal cord injury. Spinal Cord, 42, 1-6.

Kujirai, K., Kujirai, T., Sinkjaer, T. \& Rothwell, J.C. (2006) Associative plasticity in human motor cortex during voluntary muscle contraction. $J$. Neurophysiol., 96, 1337-1346.

Lamy, J.C., Russmann, H., Shamim, E.A., Meunier, S. \& Hallett, M. (2010) Paired associative stimulation induces change in presyaptic inhibition of Ia terminals in wrist flexors in humans. J. Neurophysiol., 104, 755764.

Markram, H., Lubke, J., Frotscher, M. \& Sakmann, B. (1997) Regulation of synaptic efficacy by coincidence of postsynaptic APs and EPSPs. Science, 275, 213-215.

Meunier, S., Russmann, H., Simonetta-Moreau, M. \& Hallett, M. (2007) Changes in spinal excitability after PAS. J. Neurophysiol., 97, 3131-3135.

Nielsen, J. \& Petersen, N. (1995a) Changes in the effect of magnetic brain stimulation accompanying voluntary dynamic contraction in man. J. Physiol., 484, 777-789.

Nielsen, J. \& Petersen, N. (1995b) Evidence favouring different descending pathways to soleus motoneurones activated by magnetic brain stimulation in man. J. Physiol., 486, 779-788.

Nielsen, J., Petersen, N., Deuschl, G. \& Ballegaard, M. (1993) Task-related changes in the effect of magnetic brain stimulation on spinal neurones in man. J. Physiol., 471, 223-243.

Pascual-Leone, A., Valls-Sole, J., Wassermann, E.M. \& Hallett, M. (1994) Responses to rapid-rate transcranial magnetic stimulation of the human motor cortex. Brain, 117 (Pt 1), 847-858.

Petersen, N., Christensen, L.O., Morita, H., Sinkjaer, T. \& Nielsen, J. (1998) Evidence that a transcortical pathway contributes to stretch reflexes in the tibialis anterior muscle in man. J. Physiol., 512 (Pt 1), 267-276.

Roy, F.D., Yang, J.F. \& Gorassini, M.A. (2010) Afferent regulation of leg motor cortex excitability after incomplete spinal cord injury. J. Neurophysiol., 103, 2222-2233.

Schubert, M., Beck, S., Taube, W., Amtage, F., Faist, M. \& Gruber, M. (2008) Balance training and ballistic strength training are associated with taskspecific corticospinal adaptations. Eur. J. Neurosci., 27, 2007-2018.

Song, S., Miller, K.D. \& Abbott, L.F. (2000) Competitive Hebbian learning through spike-timing-dependent synaptic plasticity. Nat. Neurosci., 3, 919926.

Stefan, K., Kunesch, E., Cohen, L.G., Benecke, R. \& Classen, J. (2000) Induction of plasticity in the human motor cortex by paired associative stimulation. Brain (Pt 3), 123, 572-584.

Stefan, K., Kunesch, E., Benecke, R., Cohen, L.G. \& Classen, J. (2002) Mechanisms of enhancement of human motor cortex excitability induced by interventional paired associative stimulation. J. Physiol., 543, 699-708.

Taube, W., Schubert, M., Gruber, M., Beck, S., Faist, M. \& Gollhofer, A. (2006) Direct corticospinal pathways contribute to neuromuscular control of perturbed stance. J. Appl. Physiol., 101, 420-429.

Taube, W., Lundbye-Jensen, J., Gollhofer, A., Schubert, M. \& Leukel, C. (2011) Evidence that the cortical motor command for the initiation of dynamic plantarflexion consists of excitation followed by inhibition. PLOS ONE, 6, e25657.

Taylor, J.L. \& Gandevia, S.C. (2004) Noninvasive stimulation of the human corticospinal tract. J. Appl. Physiol., 96, 1496-1503.

Taylor, J.L. \& Martin, P.G. (2009) Voluntary motor output is altered by spiketiming-dependent changes in the human corticospinal pathway. J. Neurosci., 29, 11708-11716.

Taylor, J.L., Todd, G. \& Gandevia, S.C. (2006) Evidence for a supraspinal contribution to human muscle fatigue. Clin. Exp. Pharmacol. Physiol., 33, 400-405.

Thompson, A.K., Lapallo, B., Duffield, M., Abel, B.M. \& Pomerantz, F. (2011) Repetitive common peroneal nerve stimulation increases ankle dorsiflexor motor evoked potentials in incomplete spinal cord lesions. Exp. Brain Res., 210, 143-152.

Ugawa, Y., Uesaka, Y., Terao, Y., Hanajima, R. \& Kanazawa, I. (1997) Magnetic stimulation of the descending and ascending tracts at the foramen magnum level. Electroencephalogr. Clin. Neurophysiol., 105, $128-131$.

Valls-Sole, J., Alvarez, R. \& Tolosa, E.S. (1994) Vibration-induced presynaptic inhibition of the soleus $\mathrm{H}$ reflex is temporarily reduced by cortical magnetic stimulation in human subjects. Neurosci. Lett., 170, 149-152.

Wolpaw, J.R. (2010) What can the spinal cord teach us about learning and memory? Neuroscientist, 16, 532-549.

Wolpaw, J.R. \& Kaas, J.H. (2001) Taking sides: corticospinal tract plasticity during development. Neurology, 57, 1530-1531. 